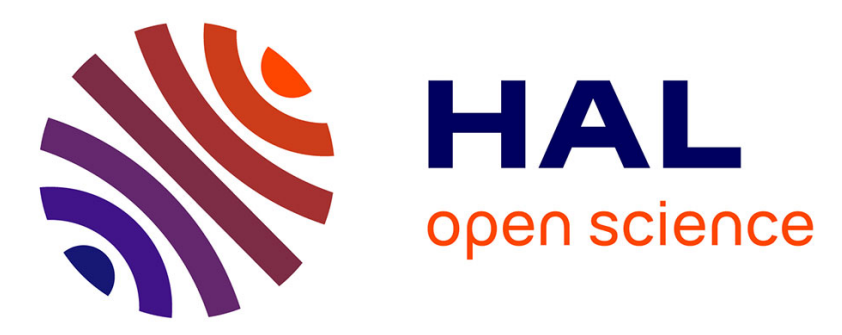

\title{
Spatially Regularized Multi-Exponential Transverse Relaxation Times Estimation From Magnitude Magnetic Resonance Images Under Rician Noise
}

Christian El Hajj, Saïd Moussaoui, Guylaine Collewet, Maja Musse

\section{- To cite this version:}

Christian El Hajj, Saïd Moussaoui, Guylaine Collewet, Maja Musse. Spatially Regularized MultiExponential Transverse Relaxation Times Estimation From Magnitude Magnetic Resonance Images Under Rician Noise. 26th IEEE International Conference on Image Processing, Sep 2019, Tapei, Taiwan. hal-02317863

\section{HAL Id: hal-02317863 \\ https://hal.science/hal-02317863}

Submitted on 16 Oct 2019

HAL is a multi-disciplinary open access archive for the deposit and dissemination of scientific research documents, whether they are published or not. The documents may come from teaching and research institutions in France or abroad, or from public or private research centers.
L'archive ouverte pluridisciplinaire HAL, est destinée au dépôt et à la diffusion de documents scientifiques de niveau recherche, publiés ou non, émanant des établissements d'enseignement et de recherche français ou étrangers, des laboratoires publics ou privés. 


\title{
SPATIALLY REGULARIZED MULTI-EXPONENTIAL TRANSVERSE RELAXATION TIMES ESTIMATION FROM MAGNITUDE MAGNETIC RESONANCE IMAGES UNDER RICIAN NOISE
}

\author{
Christian EL HAJJ ${ }^{1,2}$, Saïd MOUSSAOUI ${ }^{1}$, Guylaine COLLEWET ${ }^{2}$ and Maja MUSSE ${ }^{2}$ \\ 1: LS2N, École Centrale de Nantes, 2: Irstea, UR OPAALE, Rennes and Univ Bretagne Loire, France
}

\begin{abstract}
The extraction of multi-exponential decay parameters from multi-temporal images corrupted with Rician noise and with limited time samples proves to be a challenging problem frequently encountered in clinical and food MRI studies. This work aims at proposing a method for the estimation of multiexponential transverse relaxation times from noisy magnitude MRI images. A spatially regularized Maximum-Likelihood estimator accounting for the Rician distribution of the noise is introduced. To deal with the large-scale optimization problem, a Majoration-Minimization approach coupled with an adapted non-linear least squares algorithm is implemented. The proposed algorithm is numerically fast, stable and leads to accurate results. Its effectiveness is illustrated by an application to a simulated phantom and to magnitude multi spin echo MRI images acquired from a tomato sample.
\end{abstract}

Index Terms - Maximum-Likelihood, Spatial Regularization, Majoration-Minimization, Rician noise, Multi-T2.

\section{Introduction}

Multiple Spin Echo (Multi-SE) is a technique used in MRI transverse relaxation $\left(T_{2}\right)$ relaxometry. Multiple $T_{2}$-weighted images, at a fixed sampling rate, are acquired and an exponential decay curve is fitted to the signal at each voxel, in order to extract the relaxation times $T_{2}$ (time constants of the decay curve) and their corresponding amplitude $A_{0}$ [1]. The measured decay curve in each voxel can be associated, either to a mono-exponential decay, or, in more general cases, to a multi-exponential decay. In the latter case, extracting all the parameters describing a multi-exponential signal inside each voxel can provide relevant information on the micro-structure or composition of the tissue. In plant tissues, the components of the multi-exponential signal are assigned to principal cell compartments (vacuole, cytoplasm and wall) [2]. $T_{2}$ and $A_{0}$ maps can therefore be used to access, respectively, information about water status and distribution at the sub-cellular level, representing a smaller scale than the voxel size. This is

Thanks to Region Bretagne, École centrale Nantes, Irstea Rennes and GDR ISIS for funding. useful for evaluating cell and tissue structure and functioning, as for example in the study of the mechanical properties of the apple fruit [3]. In the brain [4], three major components can be identified within one voxel: water trapped between the myelin, the intra/extracellular water and the cerebrospinal fluid.

The $T_{2}$ values inside each voxel can be modeled either by a discretization of a continuous $T_{2}$ distribution [5], or by imposing a defined number of $T_{2}$ components $[6,7]$. We will focus our study to the second case, assuming that the number of $T_{2}$ decay components is known.

The acquisition of MRI data is carried out in the complex domain, where the real and the imaginary signal components are corrupted by an independent and identically distributed Gaussian noise. Due to various factors, such as magnetic field inhomogeneity, thermal noise, eddy currents, the phase of the complex data presents both time and space dependence. As a consequence, parameters estimation in the complex domain becomes a challenging problem. The common approach used for estimating $T_{2}$ and $A_{0}$ maps consists of taking the magnitude of the complex data. In the case of single coil acquisitions [8], the noise in the magnitude data is described by a Rician distribution, which, when not properly accounted for, introduces a bias on the estimated parameters [9].

A maximum Likelihood (ML) estimator based on the Rician probability density function can be adopted for the estimation [10]. Moroever, spatial regularization has been recently proposed to stabilize the solution by taking advantage of low voxel to voxel variability between different image structures [5, 7]. However, direct implementation of spatial regularization has not been possible because of the large amount of memory needed and the considerable computation time. It has been carried out only under Gaussian noise assumption either by dividing the image into overlapping patches [5] either by carrying the estimation on regions of interest.

In this paper, we propose an efficient algorithm which allows to carry out the estimation on the whole image at once under Rician noise assumption. In order to minimize the effect of the Rician noise we employed a ML estimator and added a penalizing function allowing us to take advantage of spatial regularity and further reduce the effect of the noise. 
The first contribution is to carry out the estimation using a Majorization-Minimization (MM) approach coupled with a Levenberg-Marquardt (LM) algorithm adapted to the estimation of all the image parameters simultaneously. The second contribution is to adopt the Armijo linesearch technique in order to ensure the convergence of the algorithm and a bounded maximal step-length to guarantee the parameters positivity. Tests were conducted on simulated phantom and on MRI images of fruit. The performance of the method is illustrated through numerical and visual results.

\section{Problem statement}

MRI data, measured with a Multi-SE sequence, is composed of $N_{\tau}$ images, of size $\left[N_{x} \times N_{y}\right]$ ( $N_{v}$ voxels). The minimum time $\triangle T E$ between two successive signal samples $\tau_{t}$ and $\tau_{t+1}$, also called echoes, depends on the image acquisition sequence. The measured signal $y_{j}\left(\tau_{t}\right)$ in each voxel $j$, can be represented by a multi-exponential decay model:

$$
M\left(\tau_{t}, \boldsymbol{\theta}_{\boldsymbol{j}}\right)=\sum_{c=1}^{N_{c}} A_{0_{(c, j)}} e^{-\frac{\tau_{t}}{T_{2}(c, j)}}, \quad \text { for } t=1, \ldots, N_{\tau}
$$

where $N_{\tau}$ represents the number of echoes and the vector $\boldsymbol{\theta}_{\boldsymbol{j}}=\left[A_{0_{(1, j)}}, T_{2_{(1, j)}} \ldots A_{0_{\left(N_{c}, j\right)}}, T_{2_{\left(N_{c}, j\right)}}\right]$ the vector of unknown parameters of length $N_{p}=2 N_{c}$. $N_{c}$ represents the number of $T_{2}$ relaxation times that corresponds to different signal components inside each voxel. By accounting for the Rician probability density function $P_{R}$, the Maximum Likelihood estimator is obtained by maximizing the following likelihood function:

$$
\begin{aligned}
L(\boldsymbol{\theta}) & =\prod_{j=1}^{N_{v}} \prod_{t=1}^{N_{\tau}} P_{R}\left(y_{j}\left(\tau_{t}\right) \mid M\left(\tau_{t}, \boldsymbol{\theta}_{j}\right), \sigma\right)= \\
& \prod_{j=1}^{N_{v}} \prod_{t=1}^{N_{\tau}} \frac{y_{j}\left(\tau_{t}\right)}{\sigma^{2}} e^{-\left[\frac{y_{j}\left(\tau_{t}\right)^{2}+M\left(\tau_{t}, \boldsymbol{\theta}_{j}\right)^{2}}{2 \sigma^{2}}\right]} I_{0}\left(\Delta_{j t}\right),
\end{aligned}
$$

where $\boldsymbol{\theta}=\left[\begin{array}{lll}\boldsymbol{\theta}_{\mathbf{1}} & \ldots & \boldsymbol{\theta}_{\boldsymbol{j}}\end{array}\right], I_{0}$ is the modified Bessel function of the first kind and order 0 and $\sigma$ is the standard deviation of the Gaussian noise in the real and imaginary parts. $\Delta_{j t}=\frac{y_{j}\left(\tau_{t}\right) M\left(\tau_{t}, \boldsymbol{\theta}_{j}\right)}{\sigma^{2}}$. At high SNR, the Rician probability function approaches the Gaussian distribution. However, low SNR inevitably occurs in relaxometry for high values of $\tau_{t}$ which implies the need to take the Rician distribution into account. In the background region no signal is present, the Rician distribution can be approximated by the Rayleigh distribution and $\sigma$ can thus be estimated using the mean value of the voxels in this region [11].

Maximizing the log of the likelihood function expressed by equation (2) comes down to minimizing the ML criterion given by:

$$
J_{M L}(\boldsymbol{y}, \boldsymbol{\theta})=\sum_{j=1}^{N_{v}} \sum_{t=1}^{N_{\tau}}\left[\frac{M\left(\tau_{t}, \boldsymbol{\theta}_{\boldsymbol{j}}\right)^{2}}{2 \sigma^{2}}-\log \left(I_{0}\left(\Delta_{j}\right)\right)\right],
$$

where $\boldsymbol{y}$ is the vector containing the measured signals for all the voxels. We can consider that there exists some similarity from one voxel to its neighbor. Spatial regularity can be accounted for by adding a penalizing function to the $J_{M L}$ criterion. For each voxel $j$ we define a neighboring voxels set $V_{j}$ (for example using a window of $3 \times 3$ voxels) and impose a penalty function $\psi$ on the difference between the parameters:

$$
J_{\text {reg }}(\boldsymbol{\theta})=\sum_{p=1}^{N_{p}} \beta(p) \sum_{j=1}^{N_{v}} \sum_{k \in V_{j}} \psi\left(\theta_{j}(p)-\theta_{k}(p)\right) .
$$

Here $\boldsymbol{\beta}$ is a weight vector attributed to the parameters of the penalty function to balance the minimization with the general estimation criterion. By adding the penalizing term to the ML criterion we obtain the following penalized ML criterion:

$$
J_{P M L}(\boldsymbol{y}, \boldsymbol{\theta})=J_{M L}(\boldsymbol{y}, \boldsymbol{\theta})+J_{\text {reg }}(\boldsymbol{\theta}) .
$$

\section{Proposed algorithm}

There exists no analytical solution for estimating the parameters using the penalized ML criterion, hence, iterative approach must be adopted. The algorithm that we are proposing in this section allows the use of the Levenberg-Marquardt (LM) algorithm in order to minimize $J_{P M L}$ criterion by adopting a MM approach. The MM algorithm is based on a technique that can be used in order to solve differential optimization problems by using a quadratic surrogate function. Let us consider a convex objective function $C(\boldsymbol{x})$, at each iteration $i$ of the MM algorithm, there exists a function Maj $\left(\boldsymbol{x} \mid \boldsymbol{x}^{(i)}\right)$ tangent to $C(\boldsymbol{x})$ at point $\boldsymbol{x}^{(i)}$ such as $C(\boldsymbol{x})$ is smaller than $\operatorname{Maj}\left(\boldsymbol{x} \mid \boldsymbol{x}^{(i)}\right)$ for all the values of $\boldsymbol{x}$ [12]. $\operatorname{Maj}\left(\boldsymbol{x} \mid \boldsymbol{x}^{(i)}\right)$ is called a tangent majorant of $C(\boldsymbol{x})$. By using this approach, the minimization of a non quadratic, non convex and multidimensional criterion can be transformed into quadratic criteria.

Actually, the $J_{M L}$ criterion is the sum of two terms, a strictly convex quadratic function and a function which is proven to be strictly concave $-\log \left(I_{0}\left(\Delta_{j}\right)\right)$ [13]. A strictly concave function can be majorized by its tangent. Thus:

$$
\begin{aligned}
& -\log \left(I_{0}\left(\Delta_{j t}^{(i)}\right)\right)-\frac{y_{j}\left(\tau_{t}\right)}{\sigma^{2}} R\left(\Delta_{j t}^{(i)}\right)\left(M\left(\tau_{t}, \boldsymbol{\theta}_{j}\right)-M\left(\tau_{t}, \boldsymbol{\theta}_{j}^{(i)}\right)\right) \\
& \geq-\log \left(I_{0}\left(\Delta_{j t}\right)\right)
\end{aligned}
$$

where $R(x)=\frac{I_{1}(x)}{I_{0}(x)}$. The quadratic ML criterion to be minimized at step $i$ of the MM algorithm can be expressed as:

$$
S_{M L}^{(i)}(\boldsymbol{\theta})=\frac{1}{2 \sigma^{2}} \sum_{j=1}^{N_{v}} \sum_{t=1}^{N_{\tau}}\left(y_{j}\left(\tau_{t}\right) R\left(\Delta_{j}^{(i)}\right)-M\left(\tau_{t}, \boldsymbol{\theta}_{j}\right)\right)^{2}
$$


Moreover, by adding the spatial regularization, the voxel wise independence of the solution is lost. We propose to use the MM algorithm in order to obtain a voxel wise separable regularization criterion. Based on Erdogan and Fesslers's work [14], if we consider a convex penalizing function $\psi$, we can compute a majorant function that reestablishes the variable separability. For each iteration $i$ of the MM algorithm we can establish the following relation $[14,15,16]$ :

$$
\begin{aligned}
& \psi\left(z_{j}-z_{k}\right)= \\
& \psi\left(\frac{1}{2}\left(2 z_{j}-\left(z_{j}^{(i)}+z_{k}^{(i)}\right)\right)+\frac{1}{2}\left(-2 z_{k}+\left(z_{j}^{(i)}+z_{k}^{(i)}\right)\right)\right) \\
& \leq \frac{1}{2}\left[\psi\left(2 z_{j}-\left(z_{j}^{(i)}+z_{k}^{(i)}\right)\right)+\psi\left(2 z_{k}-\left(z_{j}^{(i)}+z_{k}^{(i)}\right)\right)\right]
\end{aligned}
$$

for $z \in\left[\theta(1), . ., \theta\left(N_{p}\right)\right]$. The majorant function is thus a sum of the two initial convex functions $\psi$ that are now voxel wise independent at each iteration $i$ of the MM algorithm. We can thus construct a new penalizing criterion by taking advantage of the symmetry of the majorant function:

$$
\begin{aligned}
& S_{\text {reg }}^{(k)}(\boldsymbol{\theta})= \\
& \sum_{p=1}^{n_{p}} \beta(p) \sum_{j=1}^{N_{v}} \sum_{k \in V_{j}} \psi\left(2 \theta_{j}(p)-\left(\theta_{j}^{(i)}(p)+\theta_{k}^{(i)}(p)\right)\right)
\end{aligned}
$$

We propose to minimize $S_{P M L}^{(k)}(\boldsymbol{\theta})=S_{M L}^{(k)}(\boldsymbol{\theta})+S_{\text {reg }}^{(k)}(\boldsymbol{\theta})$ criterion by using the LM algorithm. It is an iterative estimation method that interchanges between the gradient descent method and the Gauss-Newton method. At each iteration $l$ of the LM algorithm the parameters are updated in the direction $d_{L M}$ that is a function of the residual $r$ and the Jacobian matrix $\boldsymbol{J}$ given by :

$$
\boldsymbol{d}_{L M}^{(l)}=\left[\boldsymbol{J}^{(l)^{T}} \boldsymbol{J}^{(l)}+\boldsymbol{\lambda}^{(l)}\right]^{-1} \boldsymbol{J}^{(l)^{T}} \boldsymbol{r}^{(l)}
$$

$\boldsymbol{r}$ is expressed such that $S_{P M L}^{(i)}(\boldsymbol{\theta})=\frac{1}{2}\|\boldsymbol{r}\|_{2}^{2} . \boldsymbol{\lambda}$ is a diagonal matrix that contains the LM parameters for each voxel. It is updated at each iteration, with an initial value that is large enough to take a small step in the steepest descent direction. As the residue $\boldsymbol{r}$ gets smaller, $\boldsymbol{\lambda}$ is decreased and the LM update direction approaches that of the Gauss-Newton algorithm. Once the direction of descent is obtained, we adopt a backtracking technique based on the Armijo condition in order to compute the step size per voxel $j$ that ensures a sufficient descent and the positivity of the parameters. In the LM algorithm the heaviest computation step is the inversion of the first term in equation (10).

Actually, by applying the LM algorithm on the whole image at once, we obtain a Jacobian of size $N_{\tau} \times\left(N_{p} N_{v}\right)$, thus a matrix of size $N_{p} N_{v} \times N_{p} N_{v}$ to inverse. However, by using the MM approach, the parameters are independent from voxel to another, thus, this matrix is block diagonal. By taking advantage of this structure, we adopt the inversion of $N_{v}$ blocks of size $N_{p} \times N_{p}$. The proposed Minimization-Majorization for the Penalized Maximum Likelihood (MM-PML) approach is summarized by the following algorithm:

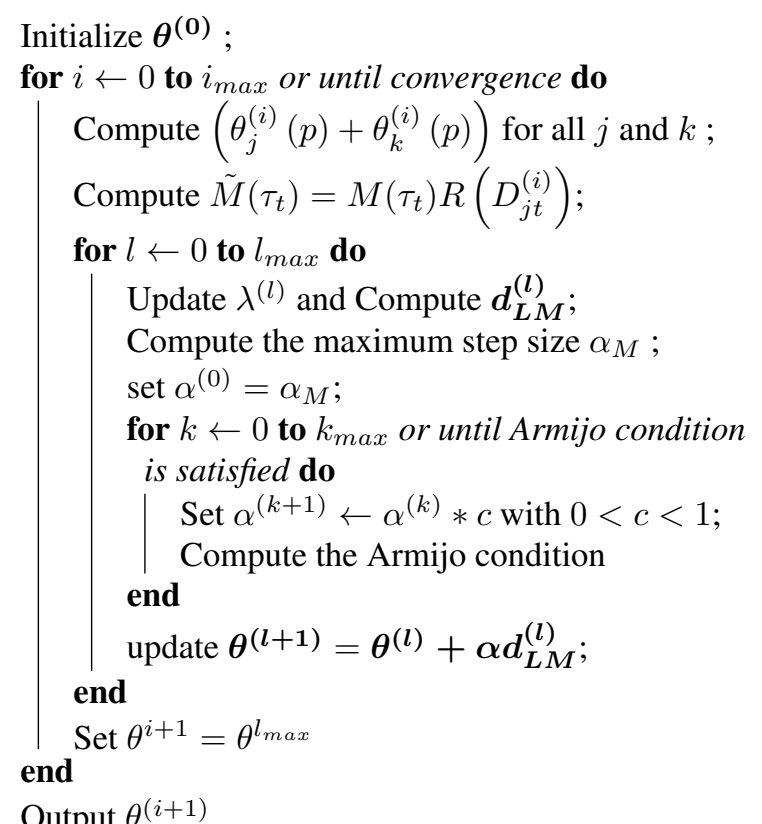

Algorithm 1: The MM algorithm for the penalized approach

\section{Results}

The evaluation was divided into two parts, the first one is based on a simulated phantom and the second one consisted in in applying the algorithm on experimental tomato MRI data. In order to simulate a phantom, a circular phantom was constructed, on an image of $128 \times 128$ voxels, with an outside ring and 9 inner disks with different sizes. A tri-exponential model was generated for the different parts of the phantom with settings that are close to parameters typically found in tomato fruits as seen in figure 5. We then added a Rician noise with a $\sigma=7$ in order to obtain a SNR value close to that found in MRI images.

For the experimental MRI settings, we used a MultiSE sequence on a 1.5T MRI scanner (Magnetom, Avanto, Siemens, Erlangen, Germany), with inter-echo spacing $(\Delta \mathrm{TE})$ of $6.5 \mathrm{~ms}$, bandwidth of $260 \mathrm{~Hz} /$ pixel, 512 echoes per echo train and a repetition time of $10 \mathrm{~s}$. The median planes of fruit (transverse section at middle height of fruit) were imaged with a total of $128 \times 128$ voxels and a slice thickness of $5 \mathrm{~mm}$, resulting in voxel size of $1.19 \times 1.19 \times 5 \mathrm{~mm}^{3}$. Two scans were made, the first one with 32 accumulations in order to obtain higher SNR and to serve as a reference, and the second one with only 1 accumulation in order to verify the stability of the algorithm at the lowest SNR.

In order to implement the regularization, we had to choose the penalizing function $\psi$ and the penalizing weight vector $\boldsymbol{\beta}$. 


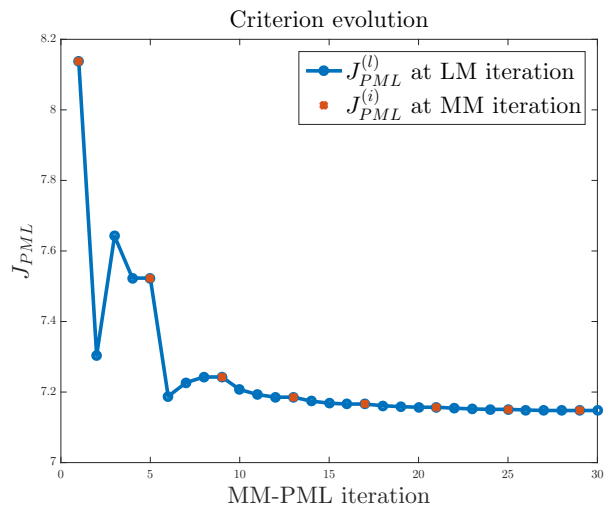

Fig. 4. The evolution of the $J_{P M L}$ criterion (in blue) across the external MM steps (orange points) and the internal LM steps (blue points)

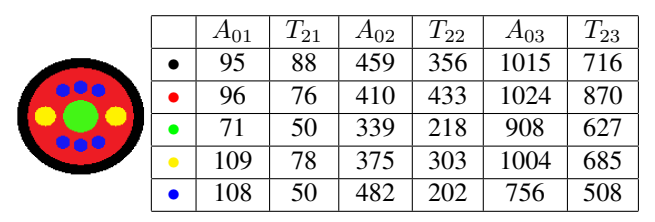

Fig. 5. $T_{2}$ and $A_{0}$ values used for generating the phantom.

The choice of the penalizing function affects the inter-tissues smoothness, whilst the penalizing weight affects the degree of smoothness on the whole image. In this study, we used an L1-L2 penalizing function with a weight $\boldsymbol{\beta}$ chosen by trial and error so as to preserve a good separation between edges. As seen in figure 1, the results on the phantom show that the reconstruction was made with satisfying edge preserving and that using the proposed MM-PML method allows to detect the different structures even in low contrast situations. For a sake of comparison the non-regularised version $(\boldsymbol{\beta}=0)$ is also represented. This illustrates the importance of the regularization without which the maps were very noisy with even no contrast between certain regions. Furthermore, we obtained a normalized root mean square error (NRMSE) of $4.47 \%$ per voxel and per parameter compared to a value of $22.85 \%$ for the non regularized version. The NRMSE was computed as: $N R M S E=100 \sqrt{\frac{1}{N_{v} N_{p}} \sum_{j=1}^{N_{v}} \sum_{p=1}^{N_{p}} \frac{\left(\theta_{j}(p)-\theta_{j}^{*}(p)\right)^{2}}{\theta_{j}^{*}(p)^{2}}}$, with $\theta_{j}^{*}(p)$ the reference parameters at voxel $j$ for parameter $p$. In figure 2 the scan with 32 acquisitions had an SNR of 687.7 whilst the images acquired with only 1 acquisition had an SNR of 145.74 (here $S N R=\frac{\sum_{j=1}^{N v} y_{j}\left(\tau_{1}\right)}{\sigma N_{v}}$ ). We used the parameters estimated from the high SNR image as a reference in order to compute the NRMSE which was equal to $5.56 \%$. Also, visually we were able to validate the robustness of the method by comparing the reconstructed maps from low and high SNR data for the different parameters which were quite similar. The evolution of the $J_{P M L}$ criterion during each step of the MM algorithm is shown in figure, it is clearly shown that by decreasing the surrogate criterion $S_{M L}$ the descent of the majorized criterion is guaranteed which validate the choice of the majorant functions.
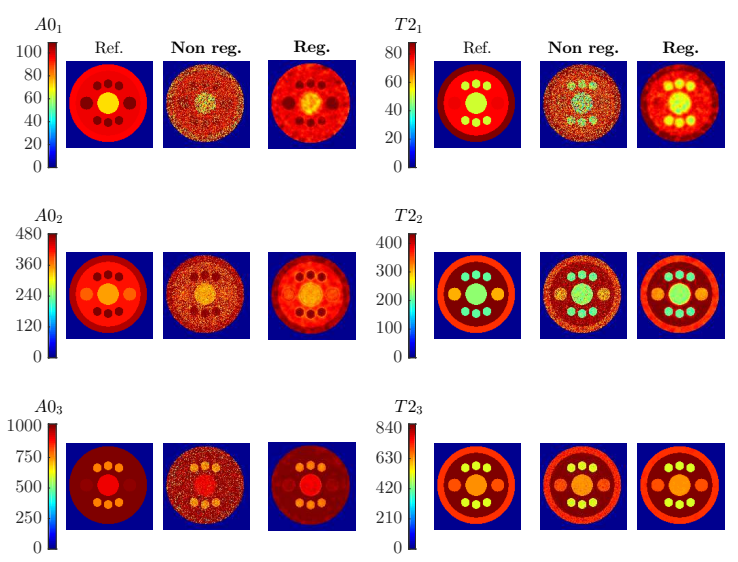

Fig. 1. Reference and estimated $A_{0}$ and $T_{2}$ maps on the phantom using a non-regularized (second columns) and the proposed regularized algorithm (third columns).

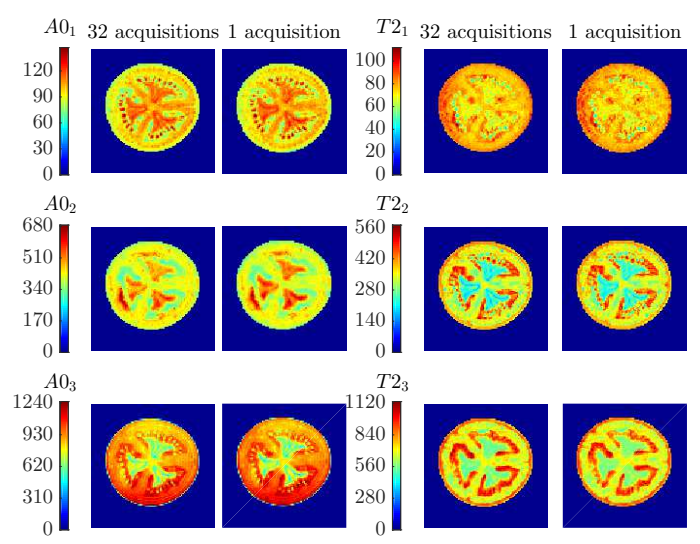

Fig. 2. $A_{0}$ and $T_{2}$ maps reconstructed from MRI images of a tomato acquired with 32 acquisitions and 1 acquisition.

\section{Conclusion}

In this paper we proposed an efficient method for the reconstruction of $T_{2}$ and $A_{0}$ maps from noisy MRI magnitude data. We showed that visually and numerically we obtained good results both on a simulated phantom and on real scans of a tomato. We were able, for the first time as far as our knowledge, to reconstruct multi-exponential maps with a voxel level information while incorperating both the Rician noise and the special regularity of the parameters. The proposed MM-PML algorithm showed both stability and accuracy. Furthermore, the estimated parameters can be used as features of a classification algorithm in order to characterize different fruit tissues. 


\section{References}

[1] H. Adriaensen, M. Musse, S. Quellec, A. Vignaud, M. Cambert, and F. Mariette, "Mse-mri sequence optimisation for measurement of bi-and tri-exponential T2 relaxation in a phantom and fruit," Magnetic resonance imaging, vol. 31, no. 10, pp. 1677-1689, 2013.

[2] Van As Henk and Van Duynhoven John, "Mri of plants and foods," Journal of Magnetic Resonance, vol. 229, pp. 25-34, 2013.

[3] M. Musse, S. Quellec, M. Cambert, M.F. Devaux, M. Lahaye, and F. Mariette, "Monitoring the postharvest ripening of tomato fruit using quantitative MRI and NMR relaxometry," Postharvest Biology and Technology, vol. 53, no. 1-2, pp. 22-35, 2009.

[4] C. Laule, I Vavasour, S Kolind, D Li, T Traboulsee, G. Moore, and A. MacKay, "Magnetic resonance imaging of myelin," Neurotherapeutics, vol. 4, no. 3, pp. 460-484, 2007.

[5] D. Kumar, T. Nguyen, S. Gauthier, and A. Raj, "Bayesian algorithm using spatial priors for multiexponential T2 relaxometry from multiecho spin echo MRI," Magnetic resonance in medicine, vol. 68, no. 5, pp. 1536-1543, 2012.

[6] M. Bouhrara, D. Reiter, H. Celik, J.M. Bonny, V. Lukas, K. Fishbein, and R. Spencer, "Incorporation of rician noise in the analysis of biexponential transverse relaxation in cartilage using a multiple gradient echo sequence at 3 and 7 Tesla," Magnetic resonance in medicine, vol. 73, no. 1, pp. 352-366, 2015.

[7] D. Hwang and Y. Du, "Improved myelin water quantification using spatially regularized non-negative least squares algorithm," Journal of Magnetic Resonance Imaging: An Official Journal of the International Society for Magnetic Resonance in Medicine, vol. 30, no. 1, pp. 203-208, 2009.

[8] R. Henkelman, "Measurement of signal intensities in the presence of noise in MR images," Medical physics, vol. 12, no. 2, pp. 232-233, 1985.

[9] J. Raya, O. Dietrich, A. Horng, J. Weber, M. Reiser, and C. Glaser, "T2 measurement in articular cartilage: impact of the fitting method on accuracy and precision at low SNR," Magnetic Resonance in Medicine: An Official Journal of the International Society for Magnetic Resonance in Medicine, vol. 63, no. 1, pp. 181-193, 2010.
[10] T. Yokoo, Q. Yuan, SéJ. négas, A. Wiethoff, and I. Pedrosa, "Quantitative R2* MRI of the liver with rician noise models for evaluation of hepatic iron overload: Simulation, phantom, and early clinical experience," Journal of Magnetic Resonance Imaging, vol. 42, no. 6, pp. 1544-1559, 2015.

[11] R. Nowak, "Wavelet-based rician noise removal for magnetic resonance imaging," IEEE Transactions on Image Processing, vol. 8, no. 10, pp. 1408-1419, 1999.

[12] D. Hunter and K. Lange, "A tutorial on MM algorithms," The American Statistician, vol. 58, no. 1, pp. 30-37, 2004.

[13] D. Varadarajan and J. Haldar, "A majorize-minimize framework for Rician and non-central chi MR images," IEEE transactions on medical imaging, vol. 34, no. 10, pp. 2191-2202, 2015.

[14] H. Erdogan and J. Fessler, "Ordered subsets algorithms for transmission tomography," Physics in Medicine \& Biology, vol. 44, no. 11, pp. 2835, 1999.

[15] A. De Pierro, "A modified expectation maximization algorithm for penalized likelihood estimation in emission tomography," IEEE transactions on medical imaging, vol. 14, no. 1, pp. 132-137, 1995.

[16] K. Lange and J. Fessler, "Globally convergent algorithms for maximum a posteriori transmission tomography," 1995. 